\title{
Assessment of DDR2, BRAF, EGFR and KRAS mutations as therapeutic targets in non-adenocarcinoma lung cancer patients
}

\author{
HIDEAKI YASHIMA $^{1,2}$, KIMIHIRO SHIMIZU $^{3}$, TAKUYA ARAKI ${ }^{1,2}$, TOHRU AOMORI $^{1,2,4}$, YOICHI OHTAKI ${ }^{3}$, \\ TOSHITERU NAGASHIMA ${ }^{3}$, YASUAKI ENOKIDA $^{3}$, JUN ATSUMI $^{3}$, TOMONORI NAKAMURA $^{1,2}$, \\ IZUMI TAKEYOSHI $^{3}$ and KOUJIROU YAMAMOTO ${ }^{1,2}$ \\ ${ }^{1}$ Department of Clinical Pharmacology, Gunma University Graduate School of Medicine, ${ }^{2}$ Department of Pharmacy, \\ Gunma University Hospital, ${ }^{3}$ Department of Thoracic and Visceral Organ Surgery, ${ }^{4}$ Center for Medical Education, \\ Gunma University Graduate School of Medicine, Maebashi, Gunma 371-8511, Japan
}

Received February 24, 2014; Accepted March 18, 2014

DOI: $10.3892 / \mathrm{mco} .2014 .302$

\begin{abstract}
Molecular-targeted therapy has not been established in non-adenocarcinoma lung cancer (non-AdLC), as no targets that affect the clinical efficacy of molecular-targeted drugs have yet been identified. In this study, we investigated the frequency of genetic variations in discoidin domain receptor 2 (DDR2), v-raf murine sarcoma viral oncogene homolog B1 (BRAF), epidermal growth factor receptor (EGFR) and v-Ki-ras2 Kirsten rat sarcoma viral oncogene homolog (KRAS) in non-AdLC patients, in order to evaluate the possibility of genetic mutations in these genes being used as therapeutic targets for the treatment of patients with non-AdLC. For this purpose, we enrolled 150 non-AdLC patients who had undergone surgery at the Gunma University Hospital between December, 2003 and December, 2012. Genetic mutations in the EGFR, KRAS, DDR2 and BRAF genes were detected by a sequencing method or probe assay using DNA derived from cancer tissues. No somatic mutations in DDR2 or BRAF were detected in non-AdLC patients. Conversely, genetic mutations in EGFR exon 19 were found in 3 squamous cell carcinoma (SCC) and 3 adenosquamous carcinoma patients, whereas KRAS codon 12 mutations were also found in 3 SCC patients and 1 large-cell neuroendocrine carcinoma patient. EGFR and KRAS mutations were mutually exclusive. This study indicated that, although DDR2 and BRAF mutations may only rarely be used as therapeutic targets, EGFR and KRAS mutations may represent candidate therapeutic targets, at least in the non-AdLC patients investigated.
\end{abstract}

Correspondence to: Dr Takuya Araki, Department of Clinical Pharmacology, Gunma University Graduate School of Medicine, 3-39-22 Showa-machi, Maebashi, Gunma 371-8511, Japan

E-mail: tkyaraki@gunma-u.ac.jp

Key words: lung cancer, non-adenocarcinoma lung cancer, epidermal growth factor receptor, v-Ki-ras2 Kirsten rat sarcoma viral oncogene homolog, discoidin domain receptor 2, v-raf murine sarcoma viral oncogene homolog B1, biomarker, molecular-targeted therapy

\section{Introduction}

Lung cancer is the leading cause of cancer-related mortality worldwide, accounting for 1.37 million deaths in 2008 (1). Lung cancer is generally treated with surgical resection, chemotherapy, radiation therapy, or combined modality approaches with curative intent. Among these treatments, chemotherapy is the only applicable and recommended treatment for patients with metastatic or relapsed lung cancer. However the clinical efficacy of conventional cytotoxic chemotherapy is insufficient, with a response rate (RR) of 20-40\% (2-4). Recently, $80 \%$ of non-smoker Asian adenocarcinoma lung cancer (AdLC) patients were found to have at least one genetic abnormality in the epidermal growth factor receptor (EGFR), v-Ki-ras2 Kirsten rat sarcoma viral oncogene homolog (KRAS), echinoderm microtubule-associated protein-like 4-anaplastic lymphoma kinase (EML4-ALK), or human EGFR-related 2 genes (5). Treatment targeting the aberrant proteins encoded by these genes has been the focus of treatment for AdLC, which accounts for $45 \%$ of lung cancer cases. In particular, patients harboring an EGFR mutation and an EML4-ALK fusion respond remarkably to the EGFR tyrosine kinase inhibitors (TKIs) erlotinib (RR, 70-83\%) or gefitinib (RR, 62-73\%) and the ALK inhibitor crizotinib (RR, 57\%), respectively (6). Thus, drugs targeting these aberrant proteins have significantly improved the treatment outcome for lung cancer patients. Similarly, an EGFR mutation is frequently encountered among patients with adenosquamous carcinoma (ASC) (7) and Iwanaga et al (8) reported a case with ASC harboring an EGFR mutation that responded significantly to EGFR-TKI treatment. Conversely, in patients with pulmonary squamous cell carcinoma (SCC), which accounts for $35 \%$ of lung cancer cases, mutations in the EGFR or KRAS genes are extremely rare $(9,10)$. Marchetti et al (9) could not find any mutations in the area encoding the tyrosine kinase domain of EGFR in 454 SCC patients. Thus, in the treatment of SCC, molecular-targeted therapy is not considered to be a suitable approach and chemotherapy is limited to the use of conventional cytotoxic antineoplastic drugs. Similarly, in other histological subtypes, such as ASC, large-cell carcinoma (LCC), large-cell neuroendocrine carcinoma (LCNEC) and small-cell lung carcinoma (SCLC), the use of molecular-targeted drugs is 
not recommended, as only a limited number of patients harbor targetable mutations (11).

Recently, the concomitant administration of dasatinib (a Src family protein TKI) and erlotinib reportedly achieved a partial response in SCC patients (12). Additionally, patients who achieve partial remission with dasatinib reportedly harbor mutations in the gene encoding discoidin domain receptor 2 (DDR2) (13), a transmembrane receptor activated by binding to extracellular collagen. In tumor cells, DDRs interrupt normal cell-matrix communication and initiate pro-migratory and proinvasive cascades (14). Hammerman et al (13) recently reported that the proliferation of SCC cell lines harboring DDR2 mutations was inhibited by dasatinib in an in vitro study and that tumors established from DDR2 mutant cell lines were highly responsive to dasatinib in a xenograft model study. Those studies indicated the possibility of dasatinib as a highly effective drug for SCC patients harboring DDR2 mutations. In a study on European and North American subjects, the frequency of mutations in DDR2 was reported to be $3.2 \%$ in SCC (13), $0.5 \%$ in AdLC (15) and 17\% in LCC (16), with no mutations identified in SCLC and LCNEC patients. In addition, DDR2 mutations were more often encountered in smokers compared to nonsmokers (17). Those studies indicated that DDR2 may be a target molecule for the treatment of non-AdLC, at least in European and North American subjects. However, ethnic differences in the frequency of DDR2 mutations were not adequately assessed, despite the presence of considerable ethnic genetic variation, such as EGFR in AdLC, which has an exceptionally high prevalence in East Asian populations (30-60\%) and a low occurrence in European and North American populations (10-20\%) (18-21). Thus, to select an appropriate therapeutic strategy, accurate data on the frequencies of genetic mutations in non-AdLC (including SCC) patients of different ethnic groups are required. In a recent study, Sasaki et al (22) failed to identify a DDR2 mutation in 166 Japanese SCC patients; however, the authors only focused on mutations in the exons encoding the receptor or transmembrane region. The available data on the frequency of DDR2 mutations are not considered sufficient to support the possible use of DDR2 mutations as a therapeutic target for SCC patients, due to the lack of data in the region encoding the kinase domain, which is considered to directly affect the clinical efficacy of dasatinib. Thus, in the present study, we investigated the frequency of mutations in DDR2 exons 5, 8 and 12-17; v-raf murine sarcoma viral oncogene homolog B1 (BRAF) V600E; EGFR exons 19 and 21; and KRAS codons 12 and 13 in Japanese non-AdLC patients to assess their potential use as therapeutic biomarkers in the Japanese population.

\section{Materials and methods}

Patients and samples. We enrolled a total of 150 patients who had undergone surgery for non-AdLC at the Gunma University Hospital (Maebashi, Gunma, Japan) between December, 2003 and December, 2012, including 87 SCC and 2 ASC patients who were previously reported by Miyamae et al (23). This study was approved by the Institutional Review Board for Clinical Trials at the Gunma University Hospital and the Ethics Committee for Human Genome Analysis of the Gunma University. Written consent was obtained from all the participants after they had been informed of the experimental procedure and the purpose of this study. The resected tumor samples were immediately frozen and stored at $-80^{\circ} \mathrm{C}$ until DNA extraction. All the tumor tissue samples were diagnosed as non-AdLC and the pathological subtype was determined by hematoxylin and eosin staining. For the cases of poorly differentiated and histologically atypical SCC or LCC and cases harboring KRAS or EGFR mutations, additional immunostaining for p63 and thyroid transcription factor 1 was performed to confirm the specific histological subtype. The cases diagnosed as SCLC or LCNEC were confirmed to exhibit neuroendocrinological differentiation by 3 types of staining for neural cell adhesion molecule, chromogranin A and synaptophysin.

Detection and characterization of mutations. DNA samples were extracted from resected tumor tissues using a QIAamp DNA mini kit (Qiagen, Hilden, Germany) and were serially diluted to $50 \mathrm{ng} / \mu \mathrm{l}$ for use as DNA templates. Mutations in EGFR exon 19 and KRAS codons 12 and 13 were detected using the Sanger sequencing method according to previous studies $(24,25)$. Mutations in EGFR exon 21 were detected by the Smart Amplification Process 2 assay, using an EGFR mutation detection kit (K.K. DNAFORM, Kanagawa, Japan). BRAF V600E mutations were detected using a LightMix kit for BRAF V600E (TIB MolBiol, Berlin, Germany). Genetic variations in 8 exons of DDR2 (exons 5, 8 and 12-17), which reportedly affect the activity of the protein or disease onset, were analyzed using the Sanger sequencing method according to previous studies, with slight modifications $(13,26)$. Briefly, amplification was performed using 0.5 U AmpliTaq Gold DNA polymerase (Applied Biosystems, Foster City, CA, USA), $2.5 \mathrm{nmol}$ of each dNTP, $1 \mathrm{X}$ polymerase chain reaction buffer, $1.8 \mathrm{nmol} \mathrm{MgCl}_{2}$, 5 pmol of each forward and reverse primer set and $25 \mathrm{ng}$ of genomic DNA template in a reaction volume of $12.5 \mu \mathrm{l}$. Polymerase chain reaction was performed with an initial $5 \mathrm{~min}$ denaturation step at $95^{\circ} \mathrm{C}$, followed by 35 cycles at $95^{\circ} \mathrm{C}$ for $30 \mathrm{sec}$, at $62^{\circ} \mathrm{C}$ for $35 \mathrm{sec}$ and at $72^{\circ} \mathrm{C}$ for $45 \mathrm{sec}$, with a final elongation step at $72^{\circ} \mathrm{C}$ for $10 \mathrm{~min}$. The annealing temperature for exon 5 was set at $66^{\circ} \mathrm{C}$ to prevent non-specific amplification.

Sequencing was performed with the Sanger dideoxy method with fluorescent automated sequencing on an ABI 3130xl Genetic Analyzer (Applied Biosystems) and the sequence data were analyzed using Sequencing Analysis Software v5.4 (Applied Biosystems).

In the patients whose cancer tissues were found to harbor genetic mutations in EGFR, KRAS, BRAF or DDR2, genomic DNA obtained from blood samples or peritumoral normal tissue was also analyzed to confirm the mutations as somatic or germline.

\section{Results}

Patient characteristics and detection of mutations. The characteristics of the patients are listed in Table I. The patients' age, gender, smoking history and histological subtypes were comparable to those of Japanese non-AdLC patients previously reported (22). Among the 150 samples, a previously unreported mutation in DDR2 (1:162748496) was identified (Table II) in exon 17 , near the area encoding the tyrosine kinase domain and resulted in an amino acid alteration (Phe804Ile). The same mutation was also detected in the samples derived from the 
Table I. Characteristics of the 150 non-adenocarcinoma patients used in this study.

\begin{tabular}{lll}
\hline Characteristics & $\begin{array}{c}\text { No. } \\
(\mathrm{n}=150)\end{array}$ & $\%$ \\
\hline Age, years (mean \pm SD) & $70.9 \pm 7.4$ & \\
Gender & & \\
Male & 129 & 86.0 \\
Female & 21 & 14.0 \\
Histological subtype & & \\
SCC & 116 & 77.33 \\
LCC & 3 & 2.00 \\
LCNEC & 11 & 7.33 \\
SCLC & 15 & 10.00 \\
ASC & 5 & 3.33 \\
Smoking history & & \\
Smoker & 144 & 96.0 \\
Never smoked & 6 & 4.0 \\
Brinkman index (mean \pm SD) & $1,175 \pm 605$ & \\
Pathological stage & & \\
IA-IB & 102 & 68.0 \\
IIA-IIB & 28 & 18.7 \\
IIIA-IV & 20 & 13.3 \\
\hline
\end{tabular}

ASC, adenosquamous carcinoma; LCC, large-cell carcinoma; LCNEC, large-cell neuroendocrine carcinoma; SCC, squamous cell carcinoma; SCLC, small-cell lung carcinoma; SD, standard deviation.

blood of each patient. Conversely, no somatic mutation of DDR2 was detected in Japanese SCC, LCC, ASC, LCNEC or SCLC patients (Table III), whereas an unreported nonsense mutation (1:162746130) was detected in 1 LCC patient (Table II). Among the LCNEC and SCLC patients, 2 different non-coding mutations, namely rs2271305 in exon 5 and rs3738807 in the upstream region of exon 13, were detected in DDR2 (Table II).

Genetic mutations of EGFR exon 19 were detected in 3 SCC and 3 ASC patients and a mutation of KRAS codon 12 in 3 SCC patients (including the cases that were previously reported by Miyamae et al) and 1 LCNEC patient (Fig. 1 and Table III) (23). The EGFR and KRAS mutations were mutually exclusive and their frequencies did not differ between central and peripheral SCCs (Table IV). BRAF V600E was not found in any of the patients (Table III).

\section{Discussion}

In a previous study on North American subjects, somatic mutations of DDR2 were detected in 3.2\% of SCC patients (13). Similar to EGFR and KRAS mutations, DDR2 mutations, particularly in the area encoding the tyrosine kinase domain, are considered to affect the intracellular downstream signaling pathways and lead to aberrant cell proliferation. In the present study, we analyzed exons 5, 8 and 12-17 of DDR2, in which somatic mutations were identified in the previous study on North American subjects; however, we did not identify a somatic
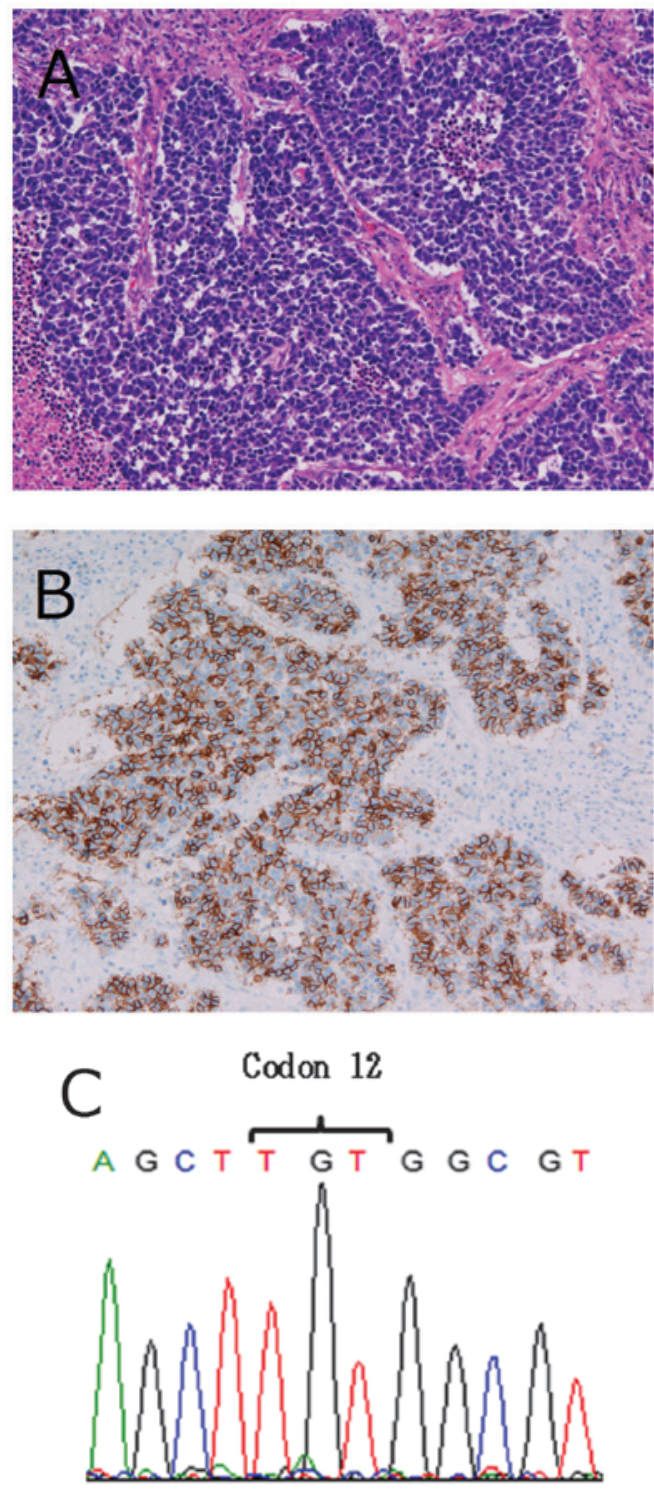

Figure 1. Histopathological staining and sequence data of a patient with large-cell neuroendocrine carcinoma harboring a v-Ki-ras2 Kirsten rat sarcoma viral oncogene homolog (KRAS) mutation. (A) Hematoxylin and eosin staining; (B) CD56 immunostaining; (C) Sequence data for KRAS codon 12.

mutation in our Japanese patients. Moreover, Sasaki et al (22) assessed the genetic diversity of exons 3-7 and 9-12 of DDR2 in Japanese patients and found no mutations. According to our results and those of Sasaki et al, we concluded that, although DDR2 is considered to be a candidate as a therapeutic target in European or North American non-AdLC patients, DDR2 mutations may only rarely be used as therapeutic targets in Japanese SCC patients, due to their extremely low frequency. Moreover, if the previously reported DDR2 mutations found in North American subjects were defined as driver mutations, our results suggested the possibility of racial differences in the oncogenic mechanism underlying SCC, since there is a racial difference in the frequency of these mutations. Similar to SCC, we were unable to identify any DDR2 mutations in other non-AdLC subtypes; however, a larger study is required to determine the frequency of DDR2 mutations in LCC, SCLC, LCNEC and ASC patients, as the sample size of our study was quite limited. 
Table II. Frequencies of DDR2 germline mutations in each histological subtype.

\begin{tabular}{|c|c|c|c|c|c|}
\hline \multirow[b]{2}{*}{ Histological subtype } & \multirow[b]{2}{*}{ No. } & \multicolumn{4}{|c|}{ Minor allele frequency (\%) } \\
\hline & & rs2271305 & rs3738807 & 1:162748496 & 1:162746130 \\
\hline $\mathrm{SCC}$ & 116 & C: 5.2 & C: 21.6 & A: 0.4 & T: 0.0 \\
\hline $\mathrm{LCC}$ & 3 & C: 0.0 & C: 16.7 & A: 0.0 & $\mathrm{~T}: 16.7$ \\
\hline LCNEC & 11 & C: 0.0 & C: 22.7 & A: 0.0 & $\mathrm{~T}: 0.0$ \\
\hline SCLC & 15 & C: 10.0 & C: 20.0 & A: 0.0 & $\mathrm{~T}: 0.0$ \\
\hline ASC & 5 & C: 10.0 & C: 40.0 & A: 0.0 & $\mathrm{~T}: 0.0$ \\
\hline All patients & 150 & C: 4.9 & C: 20.4 & A: 0.3 & T: 0.3 \\
\hline
\end{tabular}

ASC, adenosquamous carcinoma; LCC, large-cell carcinoma; LCNEC, large-cell neuroendocrine carcinoma; SCC, squamous cell carcinoma; SCLC, small-cell lung carcinoma; DDR2, discoidin domain receptor 2.

Table III. Frequencies of somatic mutations in each histological subtype.

\begin{tabular}{lrcccc}
\hline & & \multicolumn{4}{c}{ Mutation } \\
\cline { 3 - 6 } $\begin{array}{l}\text { Histological } \\
\text { subtype }\end{array}$ & No. & EGFR & KRAS & DDR2 & BRAF \\
\hline SCC & 116 & 3 & 3 & 0 & 0 \\
LCC & 3 & 0 & 0 & 0 & 0 \\
LCNEC & 11 & 0 & 1 & 0 & 0 \\
SCLC & 15 & 0 & 0 & 0 & 0 \\
ASC & 5 & 3 & 0 & 0 & 0 \\
All patients & 150 & 6 & 4 & 0 & 0
\end{tabular}

ASC, adenosquamous carcinoma; LCC, large-cell carcinoma; LCNEC, large-cell neuroendocrine carcinoma; SCC, squamous cell carcinoma; SCLC, small-cell lung carcinoma; EGFR, epidermal growth factor receptor; KRAS, v-Ki-ras2 Kirsten rat sarcoma viral oncogene homolog; DDR2, discoidin domain receptor 2; BRAF, v-raf murine sarcoma viral oncogene homolog B1.

We identified an unreported germline mutation in DDR2. Although this mutation cannot be a driver mutation, since it is congenital, it may change the clinical efficacy of dasatinib through altering the affinity of dasatinib to DDR2, as this mutation is located in the area encoding the dasatinib-binding site. Thus, a more detailed study is required to elucidate the effect of this mutation on the clinical efficacy of dasatinib.

Conversely, we identified 6 somatic mutations in EGFR and KRAS in Japanese SCC patients, despite the reportedly rare frequency of such mutations in European and North American SCC patients. Additionally, we detected EGFR mutations in 3 ASC patients and KRAS mutations in 1 LCNEC patient. To the best of our knowledge, this is first study including a LCNEC patient harboring a KRAS mutation (27). These mutations have been considered to be driver mutations and our results indicated that EGFR or KRAS inhibitors may be effective for a subset of SCC, LCNEC and ASC patients, at least in the Japanese population. Indeed, the superior clinical effect of EGFR-TKIs in Asian non-AdLC patients harboring an EGFR mutation was reported in several studies $(8,28,29)$ and molecular-targeted therapy against the downstream effectors of activated KRAS was reportedly
Table IV. Characteristics of central and peripheral squamous cell carcinoma.

\begin{tabular}{lcc}
\hline & $\begin{array}{c}\text { Central type } \\
(\mathrm{n}=25)\end{array}$ & $\begin{array}{c}\text { Peripheral type } \\
(\mathrm{n}=91)\end{array}$ \\
\cline { 2 - 3 } Mutations & No. $(\%)$ & No. $(\%)$ \\
\hline $\begin{array}{l}\text { Brinkman index } \\
\text { (mean } \pm \text { SD) }\end{array}$ & $1,357 \pm 792$ & $1,134 \pm 575$ \\
EGFR mutation & $1(4.0)$ & $2(2.2)$ \\
KRAS mutation & $1(4.0)$ & $2(2.2)$ \\
DDR2 mutation & $0(0.0)$ & $0(0.0)$ \\
BRAF mutation & $0(0.0)$ & $0(0.0)$ \\
\hline
\end{tabular}

EGFR, epidermal growth factor receptor; KRAS, v-Ki-ras2 Kirsten rat sarcoma viral oncogene homolog; DDR2, discoidin domain receptor 2; BRAF, v-raf murine sarcoma viral oncogene homolog B1; $\mathrm{SD}$, standard deviation.

more effective in patients harboring a KRAS mutation $(30,31)$. In certain studies, detection sensitivity was discussed in depth, since highly sensitive methods were required to detect somatic mutations in AdLC containing a subpopulation of mutant cells mixed within an excess of normal tissue. However, as we focused on non-AdLCs with a high cellularity of tumoral tissue and a lower percentage of normal cells compared to AdLC, we hypothesized that detection sensitivity did not affect the data.

In the present study, the frequencies of EGFR and KRAS mutations in peripheral SCCs, which are mainly encountered in the Japanese population, were not different from those in central SCCs, which are mainly encountered in European and North American populations (32). These data indicate that the difference in mutation frequency may be caused by a difference in the cancer or oncogenic pathway between Japanese and European and North American subjects, rather than by a difference in the affected site.

As candidate therapeutic targets for non-AdLCs other than DDR2, certain genetic aberrations have been reported, including fibroblast growth factor receptor 1 amplification, phosphatidylinositol-4,5-bisphosphate 3-kinase, catalytic 
subunit $\alpha$ mutations, v-akt murine thymoma viral oncogene homolog 1 mutations and phosphatase and tensin homolog mutations (33). Since our results suggested that carcinogenic genes in non-AdLCs exhibit ethnic differences, a more detailed evaluation of these candidate genes in different ethnicities is required to make more appropriate suggestions for personalized drug therapy. Furthermore, since dasatinib inhibits a number of tyrosine kinases, including DDR2 (34), the association between the expression level or mutations of tyrosine kinases and the efficacy of dasatinib requires more detailed assessment.

In conclusion, this study suggests that DDR2 mutations and the BRAF V600E mutation may only rarely be used as therapeutic targets, at least in Japanese non-AdLC patients; however, EGFR and KRAS mutations may represent candidate therapeutic targets in Japanese non-AdLC patients. The detection of EGFR and KRAS mutations may be used to select the optimal treatment for Japanese non-AdLC patients. Other genetic aberrations that may be used as therapeutic targets should be assessed in detail among different ethnic groups.

\section{Acknowledgements}

We would like to thank Ms. Aiko Matsumoto for the secretarial assistance.

\section{References}

1. Jemal A, Bray F, Center MM, Ferlay J, Ward E and Forman D: Global cancer statistics. CA Cancer J Clin 61: 69-90, 2011.

2. Scagliotti GV, Parikh P, von Pawel J, et al: Phase III study comparing cisplatin plus gemcitabine with cisplatin plus pemetrexed in chemotherapy-naive patients with advanced-stage non-small-cell lung cancer. J Clin Oncol 26: 3543-3551, 2008.

3. Schiller JH, Harrington D, Belani CP, et al: Comparison of four chemotherapy regimens for advanced non-small-cell lung cancer. N Engl J Med 346: 92-98, 2002.

4. Sandler A, Gray R, Perry MC, et al: Paclitaxel-carboplatin alone or with bevacizumab for non-small-cell lung cancer. N Engl J Med 355: 2542-2550, 2006.

5. Sun Y, Ren Y, Fang Z, et al: Lung adenocarcinoma from East Asian never-smokers is a disease largely defined by targetable oncogenic mutant kinases. J Clin Oncol 28: 4616-4620, 2010.

6. Gaughan EM and Costa DB: Genotype-driven therapies for non-small cell lung cancer: focus on EGFR, KRAS and ALK gene abnormalities. Ther Adv Med Oncol 3: 113-125, 2011.

7. Toyooka S, Yatabe Y, Tokumo M, et al: Mutations of epidermal growth factor receptor and K-ras genes in adenosquamous carcinoma of the lung. Int J Cancer 118: 1588-1590, 2006.

8. Iwanaga K, Sueoka-Aragane N, Nakamura T, Mori D and Kimura S: The long-term survival of a patient with adenosquamous lung carcinoma harboring EGFR-activating mutations who was treated with gefitinib. Intern Med 51: 2771-2774, 2012.

9. Marchetti A, Martella C, Felicioni L, et al: EGFR mutations in non-small-cell lung cancer: analysis of a large series of cases and development of a rapid and sensitive method for diagnostic screening with potential implications on pharmacologic treatment. J Clin Oncol 23: 857-865, 2005.

10. Rekhtman N, Paik PK, Arcila ME, et al: Clarifying the spectrum of driver oncogene mutations in biomarker-verified squamous carcinoma of lung: lack of EGFR/KRAS and presence of PIK3CA/AKT1 mutations. Clin Cancer Res 18: 1167-1176, 2012.

11. Lindeman NI, Cagle PT, Beasley MB, et al: Molecular testing guideline for selection of lung cancer patients for EGFR and ALK tyrosine kinase inhibitors: guideline from the College of American Pathologists, International Association for the Study of Lung Cancer, and Association for Molecular Pathology. Arch Pathol Lab Med 137: 828-860, 2013.

12. Haura EB, Tanvetyanon T, Chiappori A, et al: Phase I/II study of the Src inhibitor dasatinib in combination with erlotinib in advanced non-small-cell lung cancer. J Clin Oncol 28: 1387-1394, 2010.
13. Hammerman PS, Sos ML, Ramos AH, et al: Mutations in the DDR2 kinase gene identify a novel therapeutic target in squamous cell lung cancer. Cancer Discov 1: 78-89, 2011.

14. Valiathan RR, Marco M, Leitinger B, Kleer CG and Fridman R: Discoidin domain receptor tyrosine kinases: new players in cancer progression. Cancer Metastasis Rev 31: 295-321, 2012.

15. Ding L, Getz G, Wheeler DA, et al: Somatic mutations affect key pathways in lung adenocarcinoma. Nature 455: 1069-1075, 2008

16. Davies H, Hunter C, Smith R, et al: Somatic mutations of the protein kinase gene family in human lung cancer. Cancer Res 65: 7591-7595, 2005

17. An SJ, Chen ZH, Su J, et al: Identification of enriched driver gene alterations in subgroups of non-small cell lung cancer patients based on histology and smoking status. PLoS One 7: e40109, 2012.

18. Lynch TJ, Bell DW, Sordella R, et al: Activating mutations in the epidermal growth factor receptor underlying responsiveness of non-small-cell lung cancer to gefitinib. N Engl J Med 350: 2129-2139, 2004.

19. Paez JG, Jänne PA, Lee JC, et al: EGFR mutations in lung cancer: correlation with clinical response to gefitinib therapy. Science 304: 1497-1500, 2004.

20. Pao W, Miller V, Zakowski M, et al: EGF receptor gene mutations are common in lung cancers from 'never smokers' and are associated with sensitivity of tumors to gefitinib and erlotinib. Proc Natl Acad Sci USA 101: 13306-13311, 2004.

21. Calvo E and Baselga J: Ethnic differences in response to epidermal growth factor receptor tyrosine kinase inhibitors. J Clin Oncol 24: 2158-2163, 2006.

22. Sasaki H, Shitara M, Yokota K, et al: DDR2 polymorphisms and mRNA expression in lung cancers of Japanese patients. Oncol Lett 4: 33-37, 2012.

23. Miyamae Y, Shimizu K, Hirato J, et al: Significance of epidermal growth factor receptor gene mutations in squamous cell lung carcinoma. Oncol Rep 25: 921-928, 2011.

24. Araki T, Shimizu K, Nakamura T, et al: Clinical screening assay for EGFR exon 19 mutations using PNA-clamp smart amplification process version 2 in lung adenocarcinoma. Oncol Rep 26: 1213-1219, 2011

25. Araki T, Shimizu K, Nakamura K, et al: Usefulness of peptide nucleic acid (PNA)-clamp smart amplification process version 2 (SmartAmp2) for clinical diagnosis of KRAS codon 12 mutations in lung adenocarcinoma: comparison of PNA-clamp SmartAmp2 and PCR-related methods. J Mol Diagn 12: 118-124, 2010.

26. Ali BR, Xu H, Akawi NA, et al: Trafficking defects and loss of ligand binding are the underlying causes of all reported DDR2 missense mutations found in SMED-SL patients. Hum Mol Genet 19: 2239-2250, 2010.

27. Iyoda A, Travis WD, Sarkaria IS, et al: Expression profiling and identification of potential molecular targets for therapy in pulmonary large-cell neuroendocrine carcinoma. Exp Ther Med 2: 1041-1045, 2011

28. Tanaka K, Hata A, Kida Y, et al: Gefitinib for a poor performance status patient with squamous cell carcinoma of the lung harboring EGFR mutation. Intern Med 51: 659-661, 2012.

29. Zhou C, Wu YL, Chen G, et al: Erlotinib versus chemotherapy as first-line treatment for patients with advanced EGFR mutationpositive non-small-cell lung cancer (OPTIMAL, CTONG-0802): a multicentre, open-label, randomised, phase 3 study. Lancet Oncol 12: 735-742, 2011.

30. Jänne PA, Shaw AT, Pereira JR, et al: Selumetinib plus docetaxel for KRAS-mutant advanced non-small-cell lung cancer: a randomised, multicentre, placebo-controlled, phase 2 study. Lancet Oncol 14: 38-47, 2013.

31. Sequist LV, von Pawel J, Garmey EG, et al: Randomized phase II study of erlotinib plus tivantinib versus erlotinib plus placebo in previously treated non-small-cell lung cancer. J Clin Oncol 29: 3307-3315, 2011.

32. Sakurai H, Asamura H, Watanabe S, Suzuki K and Tsuchiya R: Clinicopathologic features of peripheral squamous cell carcinoma of the lung. Ann Thorac Surg 78: 222-227, 2004.

33. Drilon A, Rekhtman N, Ladanyi M and Paik P: Squamous-cell carcinomas of the lung: emerging biology, controversies, and the promise of targeted therapy. Lancet Oncol 13: e418-e426, 2012.

34. Montero JC, Seoane S, Ocaña A and Pandiella A: Inhibition of SRC family kinases and receptor tyrosine kinases by dasatinib: possible combinations in solid tumors. Clin Cancer Res 17: 5546-5552, 2011. 